\title{
COMPOSITE OPTICAL SPECTRA OF RADIO QUASARS
}

\author{
JOANNE C. BAKER \\ Mullard Radio Astronomy Observatory, Cavendish Laboratory, \\ Madingley Rd, Cambridge CB3 OHE, UK. \\ AND \\ RICHARD W. HUNSTEAD \\ Department of Astrophysics, School of Physics, University of \\ Sydney, NSW 2006, Australia.
}

\section{Introduction}

Primarily to illustrate the effects of orientation on the optical properties of radio-loud quasars, we have created a set of composite optical spectra (see also Baker \& Hunstead 1995). Optical spectra drawn from the $408-\mathrm{MHz}$ Molonglo Quasar Sample (MQS) have been coadded in four sets according to $R$, the ratio of radio core-to-lobe flux, which is used as an orientation indicator (eg. Orr \& Browne 1982). Compact steep-spectrum (CSS) quasars (see review by Fanti, this volume) have been combined separately, revealing for the first time many distinguishing features in their average spectra.

\section{The Composite Spectra}

Figure 1 reveals clear differences between quasars of different $R$ and the CSS quasars. A detailed comparison is made in Baker \& Hunstead (1995). In summary, we find that with decreasing $R$ (i) the optical continuum steepens, (ii) the $3000 \AA$ broad emission feature decreases in relative strength, and (iii) the narrow-line equivalent widths, broad line widths and Balmer decrements increase (Baker et al. in prep.). The above trends suggest that the nuclei of many lobe-dominated quasars are viewed through layers of dust, perhaps associated with the hazy outer regions of a torus.

The composite for the CSS quasars in the MQS is especially revealing. The average continuum slope is a very steep power law, with broad features, such as around $3000 \AA$, missing. Enhanced narrow-line emission, 


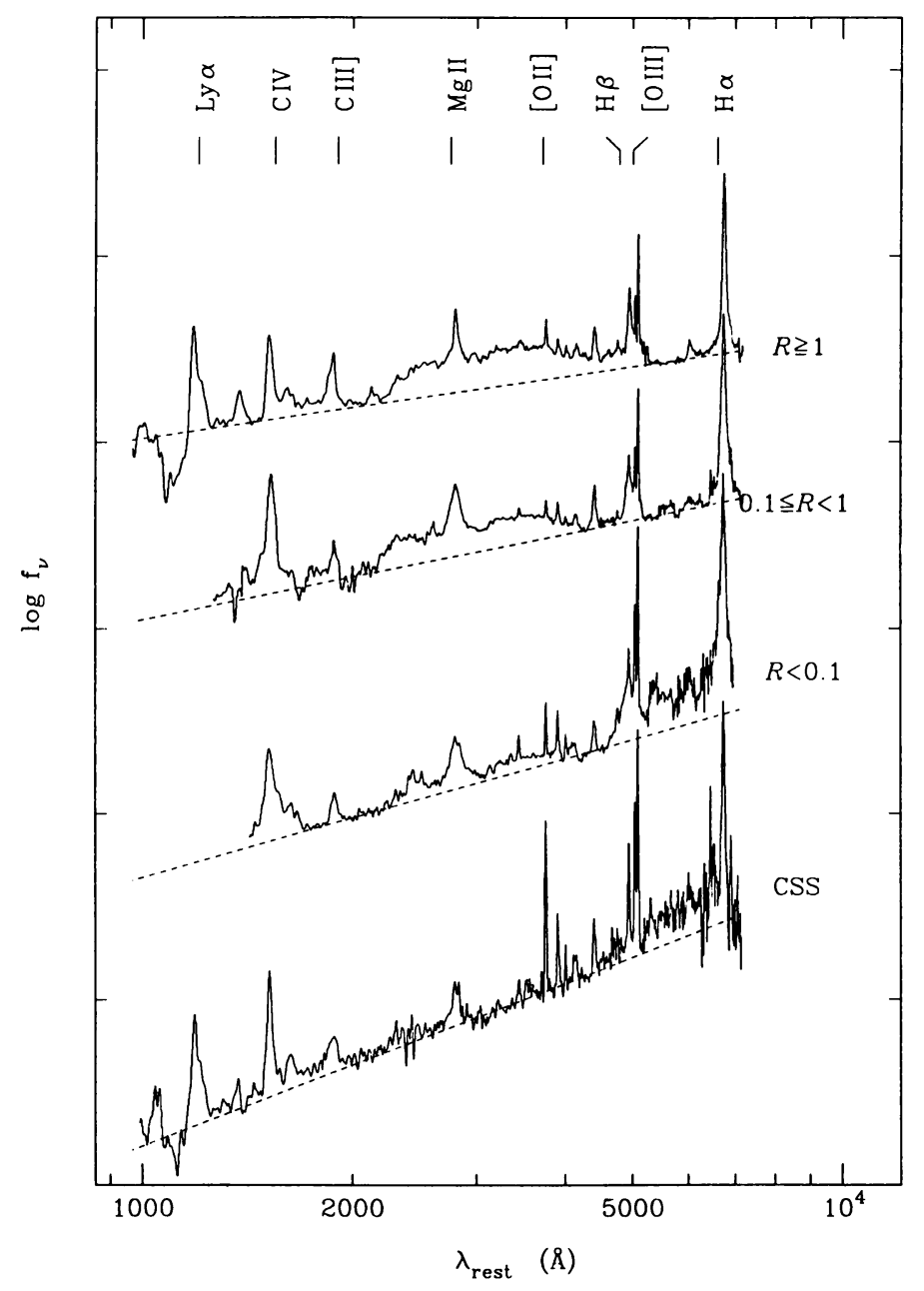

Figure 1. Composite spectra for the MQS, separated according to $R$ (see text). Best-fit power-law spectral slopes are shown as dashed lines (Baker \& Hunstead 1995).

particularly low-ionisation species, may indicate strong jet-ISM interactions. Self-absorption of $\mathrm{Mg}$ II $\lambda 2798$ and the low $\mathrm{Ly} \alpha / \mathrm{C}$ IV ratio point to heavy absorption in these sources.

\section{References}

[1] Antonucci, R.R.J. (1993), ARAA, 31, 473

[2] Baker, J.C. and Hunstead, R.W. (1995), $A p J L, 452$, L95

[3] Orr, M. and Browne, I.W.A. (1982), MNRAS, 200, 1067 